\title{
Image Pre-processing on Character Recognition using Neural Networks
}

\author{
Khushbu \\ M.Tech Research scholar \\ Computer Engineering Section \\ Maharishi Markandeshwar University \\ Haryana, India
}

\author{
Sakshi Mehta \\ Assistant Professor \\ Computer Engineering Section
}

\begin{abstract}
This paper, presents a theoretical and practical basis of preprocessing on handwritten text for character recognition using forward-feed neural networks. Afterwards, the Feed forward algorithm gives working of a neural network followed by the Back Propagation Algorithm which compromises Training, Calculating Error, and Modifying Weights. The proposed solutions focus on applying Back Propagation Algorithm model with the use of flexible pre-processing methods. The use of artificial neural network in applications can dramatically simplify the code and improve quality of recognition while achieving good performance. The relative performance evaluation of these algorithms has been also carried out based on the network noises and their connection recovered.
\end{abstract}

\section{General Terms}

\section{Methodology of Pre-processing methods using neural network}

\section{Keywords}

Image pre-processing, handwritten characters (scanned), character recognition, back propagation algorithm, network connection.

\section{INTRODUCTION}

Research in the field of pre-processing on character recognition using neural network is an improvement of the image data that suppresses unwanted distortions or enhances some image features important for further processing. Image pre-processing is the technique of enhancing data images prior to computational processing. Preprocessing is the first phase of document analysis. The purpose of preprocessing is to improve the quality of the image being processed. It makes the subsequent phases of image processing like recognition of characters easier. In the preprocessing step noise and other variations are avoidable. Image preprocessing methods use the considerable redundancy in images. This paper also shows that how the use of artificial neural network simplifies development of a character recognition application, while achieving highest quality of recognition and good performance one of the most classical applications of the Artificial Neural Network is the Character Recognition System. Because of less time consuming and cost effective property, this system have many different types of applications in various fields, many of which are following that use in daily life.

$>$ Used in businesses, post offices, banks, security surveillance systems.
$>$ In the field of robotics employ this system as the base of their operations or teaching a robot to pick up the objects.

$>$ Processing a check for various purposes.

$>$ Performing an eye/face scan at the airport entrance.

This paper brings out the standard methodology for preprocessing methods using the performance evaluation of neural network algorithm in an efficient way. Section 2 discusses the related works on preprocessing of character recognition using neural network and Section 3 describe the Problem Statement that define the idea to evaluate the performance. Section 4 and 5 shows the way of standard methodology for construction model and implementation details of the network algorithm. Section 6 and 7 describe the results and conclusion theory of this network system.

\section{RELATED WORK}

Miroslav Nohaj et al [1] presents primary task of this master's is to create a theoretical and practical basis of preprocessing of printed text for optical character recognition using forwardfeed neural networks. Demonstration application was created and its parameters were set according to results of realized experiments.

Kosbatwar et al [2] define the use of artificial neural network in applications can dramatically simplify the code and improve quality of recognition while achieving good performance. Another benefit of using neural network in application is extensibility of the system - ability to recognize more character sets than initially defined. Most of traditional systems are not extensible enough. In this paper recognition of characters is possible by using neural network back propagation algorithm.

Amit Kumar Gupta et al [4] present that analyzing neural network method in pattern recognition. A neural network is a processing device, whose design was inspired by the design and functioning of human brain and their components. The proposed solutions focus on applying Back Propagation Algorithm model for pattern recognition. The primary function of which is to retrieve in a pattern stored in memory, when an incomplete or noisy version of that pattern is presented. An associative memory is a storehouse of associated patterns that are encoded in some form.

Fakhraddin Mamedov et al [5] describe the Character Recognition System in which we creating a Character Matrix and a corresponding Suitable Network Structure is key. In 
addition, knowledge of how one is Deriving the Input from a Character Matrix must first be obtained before one may proceed. Afterwards, the Feed Forward Algorithm gives insight into the enter workings of a neural network; followed by the Back Propagation Algorithm.

Sameeksha Barve [10] proposed an Optical Character recognition system based on Artificial Neural Networks (ANNs). The ANN is trained using the Back Propagation algorithm. In the proposed system, each typed English letter is represented by binary numbers that are used as input to a simple feature extraction system whose output, in addition to the input, are fed to an ANN.

\section{PROBLEM STATEMENT}

Nowadays the neural network has demonstrated its capability for solving complex character recognition problems. Commonly solved problems have limited scope. To increase the performance in some extent, after applying the preprocessing methods having more flexibility than others, the system describe the neural network model followed by the back propagation algorithm. The relative performance evaluation of this algorithm has been carried out. The evaluation of algorithm has been performed based on following criteria:

(1) Noise in weights

(2) Noise in inputs

(3) Loss of connections

\section{PROPOSED ALGORITHM}

This section will explain the proposed algorithm, i.e. what are different steps involve achieving in Image Preprocessing on Character Recognition Using Neural Network. In first step optical scanners are used, which generally consist of a transport mechanism plus a sensing device that converts light intensity into gray levels, through the scanning process a digital image of the original document is captured. The image resulting from the scanning process may contain a certain amount of noise due to defected medium or by others. In the second step describe implementation of preprocessing technique by loading the image. After loading a scan document select the character one by one. In the third step recognize the character using Back Propagation algorithm as shown in fig. 1 include following steps and structure.

The algorithm requires three major steps that define the system working theory and reason to behind their implementation.1) Image preprocessing 2) Neural network recognition 3) Back propagation Algorithm.

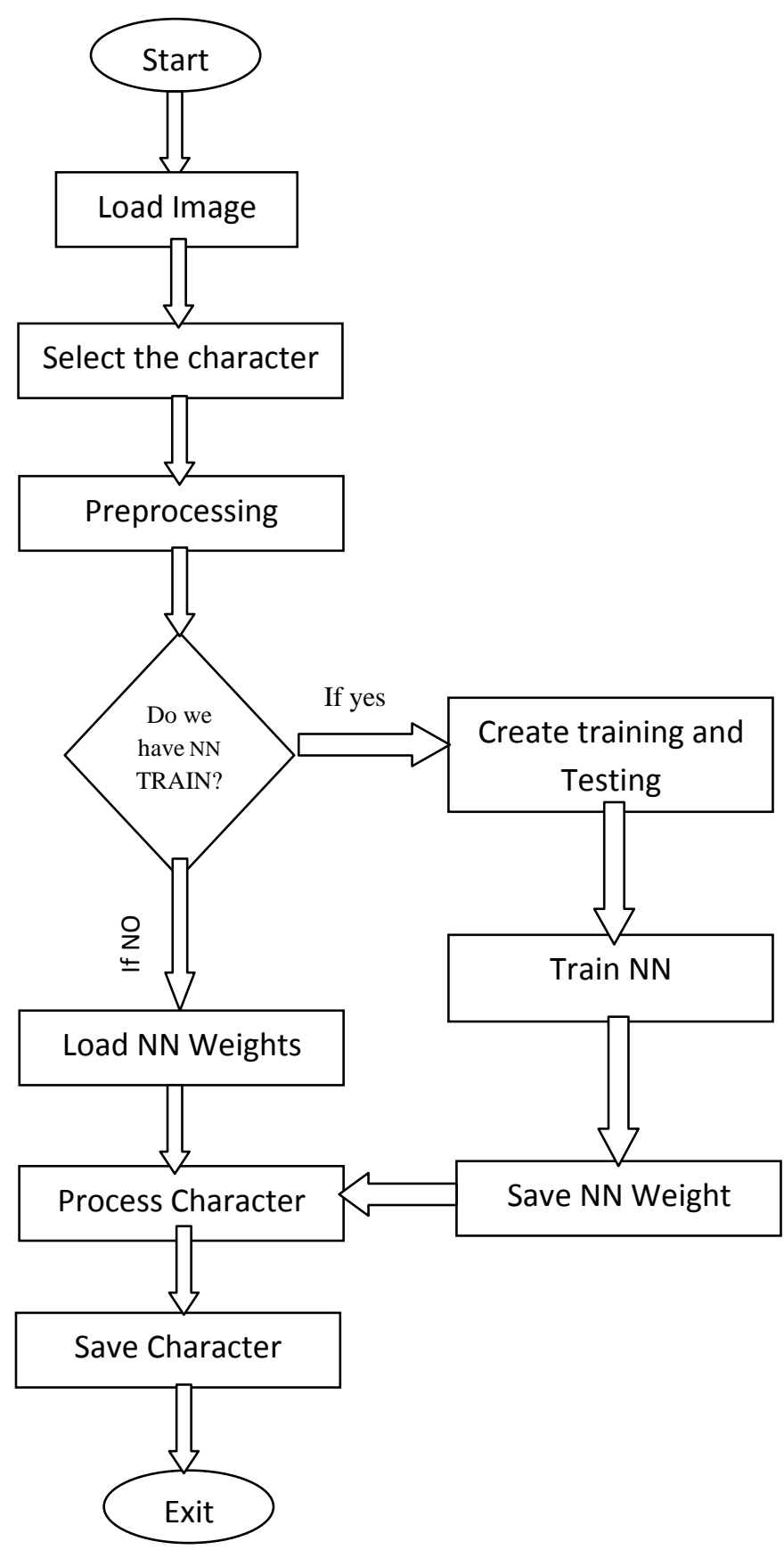

Fig 1: Proposed Algorithm

\subsection{Image Preprocessing}

Almost everyone who is working with computers has to input some text to the computer from the paper. There is not only one way to do that. The smartest way is to scan the document and let software for character recognition transform the scanned image into editable text. The Character Recognition (CR) software can use methods like: Matrix comparation of image with letter examples, Feature extraction from image, Recognition of characters using neural networks, Hybrid and combined methods, other methods. Each method listed above has some advantages and disadvantages so, during use of CR software which uses any of those methods, what can expect. In this research when input the handwritten scan Document shown in fig.2 and fig. 3. Flexibility of the methods listed 
above varies from one to another, but even the less flexible method's success can be improved using image preprocessing before the CR. The most used methods of image pre-processing before $\mathrm{CR}$ are: Thresholding based on histogram, Smoothing, Other 2D matrix filters.

\subsection{Neural Network Recognition}

One of the major applications of neural network is character recognition. An Artificial Neural Network (ANN) is an information processing paradigm that is inspired by the way biological nervous systems, such as the brain, process information. The ANN encompasses a large variety of models all of which have two important strings.

1) They are composed of a large number of structurally and functionally similar units called neurons usually connected various configurations by weighted links.

2) The Ann's model parameters are derived from supplied I/O paired data sets by an estimation process called training.

Different neural network algorithms are used for various algorithms differ in their learning mechanism. Information is stored in the weight matrix of a neural network. Learning is the determination of the weights. All learning methods used for adaptive neural networks can be classified into two major categories: supervised learning and unsupervised learning. The supervised learning method is followed by back propagation algorithm. With the use of supervised learning, how the algorithm design the network is describe in the section 4.3 .

\subsection{Back Propagation Algorithm}

The feed forward network can be applied to a variety of classification and recognition problems. In back propagation algorithm, it is not necessary to know about the mathematical model of the classification of recognition problem to train and then recall information from the network.

In this algorithm, if suitable network architecture is selected and sufficient training set is presented, the network may give acceptable solution.

The algorithm steps that are followed by the back propagation algorithm:

1. Input Data is presented to the First Layer's processing elements and then propagated along the interconnections to the first hidden layer.

2. Each processing element, $h$ in the hidden layer calculates the sum of the weights inputs for each input, i, from the previous layer.

Weighted $\operatorname{sum}_{\mathrm{h}}=\Sigma\left(\right.$ weight $_{\mathrm{h}} *$ Input $\left._{\mathrm{h}}\right)$

3. The output from the hidden layer processing element, $\mathrm{h}$, is then calculated with the activation function, $f$, and then propagated to the next layer. A typical sigmoidal activation function would be $\mathrm{f}(\mathrm{x})=1 /(1+\mathrm{e}-\mathrm{x})$.

Hidden output $=\mathrm{f}\left(\right.$ weighted sum $_{\mathrm{h}}+$ bias $\left._{\mathrm{h}}\right)$

4. Step 2-3 is repeated for each hidden layer until the output layer is reached.
5. Each processing element o, in the output layer calculates the sum of weighted input from each input $\mathrm{h}$, from the previous layer.

Weighted sumo $=\Sigma\left(\right.$ weighto $_{\mathrm{h}} *$ input $\left._{\mathrm{oh}}\right)$, for all $\mathrm{h}$

6. The output from the output layer processing element $o$, is then calculated with the activation function, $\mathrm{f}$.

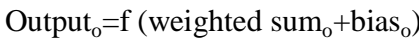

7. The calculated output is compared to the target output to compute an error for each output layer's processing element. The derivative of the activation function $\mathrm{f}$, used to find rate of change. For this e.g. $\mathrm{f}^{\prime}(\mathrm{x})=\mathrm{f}(\mathrm{x})(1-\mathrm{f}(\mathrm{x}))$.

Iteration and the network learn while iterating repeatedly until a net minimum error value is achieved.

$$
\text { Error }_{\mathrm{o}}=\left(\text { target }_{\mathrm{o}}-\text { output }_{\mathrm{o}}\right) \mathrm{f}^{\prime}\left(\text { weighted }_{\text {sum }_{\mathrm{o}}}+\text { bias }_{\mathrm{o}}\right)
$$

8. The error for the processing element in the hidden layer(s) is calculated by propagating the error back through the network. Note weighted $\mathrm{h}_{\mathrm{h}}$ is the weight of the output unit, o corresponding to the connection to the hidden unit, $\mathrm{h}$ for all o

Error $_{\mathrm{h}}=\mathrm{f}^{\prime}\left(\right.$ weighted $\operatorname{sum}_{\mathrm{h}}+$ bias $\left._{\mathrm{h}}\right) * \Sigma\left(\right.$ error $_{\mathrm{o}}{ }^{*}$ weight $\left._{\mathrm{oh}}\right)$

9. The weights in the output layer are updated, $\beta=$ learn rate constant between 1 and $0, \alpha=$ momentum constant. The term $\beta^{*}$ error $_{o}$ represents the heart of the delta rule. Input $o$ is the input value carried along the interconnection from the hidden layer to the output layer.

Weight $_{\mathrm{o}}(\mathrm{t}+1)=$ weight $_{\mathrm{o}}(\mathrm{t})+\beta *$ error $_{\mathrm{o}} *\left(\right.$ input $_{\mathrm{o}} /$ inputi 2$)+\alpha *($ weight ${ }_{o}(\mathrm{t})$. weight $\left._{\mathrm{o}}(\mathrm{t}-1)\right)$

10 The weights in each hidden layer are updated. $\beta=$ learn rate constant between 1 and $0, \alpha=$ momentum constant.

Weight $_{0}(t+1)=$ weight $_{h}(t)+\beta * \operatorname{errorh}^{*}\left(\right.$ input $_{0} /$ inputoi2 $)+\alpha *($ weig $\mathrm{ht}_{\mathrm{h}}(\mathrm{t})$. weight $\left._{\mathrm{h}}(\mathrm{t}-1)\right)$

Note: Step 1-6 constitutes the forward propagation of information flow while step 7-10 constitute the back propagation of the error.

\section{IMPLEMENTATION}

Image preprocessing is the technique of enhancing data images prior to computational processing. The way is to scan the document and let software for character recognition transform the image into editable text. The most used methods of image preprocessing before $\mathrm{CR}$ are:

$>$ Image cropping and filtering

Intensity adjustment and histogram equalization

$>$ Brightness and Thresholding

$>$ Clearing areas of binary image

$>$ Detecting edges.

After the preprocessing methods, recognize the character one by one using back propagation algorithm.

\subsection{Architecture of back propagation algorithm}

In the architecture of back propagation, network receives the 2 input neurons. It is then required to identify the letter by 
responding with a 26 -element output vector. The 26 elements of the output vector each represent a letter. To operate correctly, the network should respond with a 1 in the position of the letter being presented to the network. All other values in the output vector should be 0 . In addition, the network should be able to handle noise. In practice, the network does not receive a perfect Boolean vector as input. Specifically, the network should make as few mistakes as possible.

The network is a two-layer log-sigmoid network. The logsigmoid transfer function was picked because its output range ( 0 to 1 ) is perfect for learning to output Boolean values. If the network has trouble learning, then neurons can be added to hidden layer. The network is trained to output a 1 in the correct position of the output vector and to fill the rest of the output vector with 0's. However, noisy input vectors may result in the network not creating perfect 1's and 0's. After the network is trained the output is passed through the competitive transfer function compet. This makes sure that the output corresponding to the letter most like the noisy input vector takes on a value of 1 , and all others have a value of 0 .There are two sets of weights; input-hidden layer weights and hidden-output layer weights. These weights represent the memory of the neural network, where final training weights can be used when running the network. Initial weights are generated randomly there, after; weights are updated using the error (difference) between the actual output of the network and the desired (target) output.

To evaluate the performance, the back propagation is again divided into 3 criteria when noise is present in inputs, in weights and having connection losses. By applying the algorithm that generalizes itself, system gives the high performance. The working of the character recognition is shown in Fig -2 and Fig -3 that describe the system recognition with evaluating these three criteria. Fig shows the Recognition steps and the character after completing the recognition.

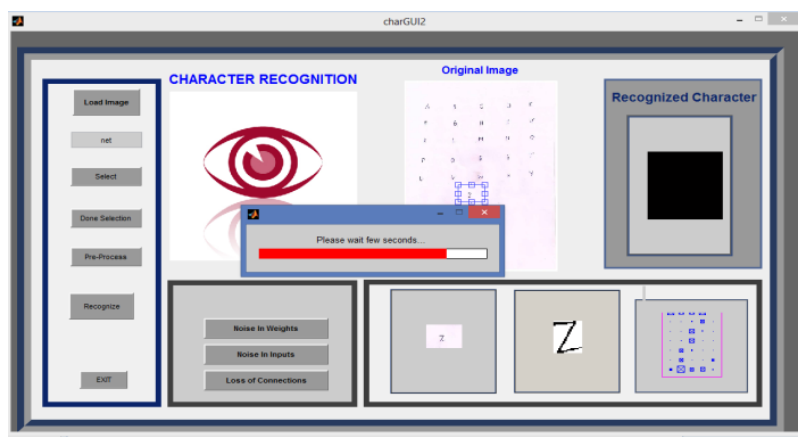

\section{CONCLUSIONS}

An approach has been made to increase the accuracy of recognition of handwritten scanned character. The neural networks can be used for image preprocessing pretty well with many advantages over normal image preprocessing methods. The results of analysis also show how the network noises and connection losses have recover with the evaluation of the back propagation algorithm during character recognition.
Fig 2: Recognition of character using back propagation algorithm

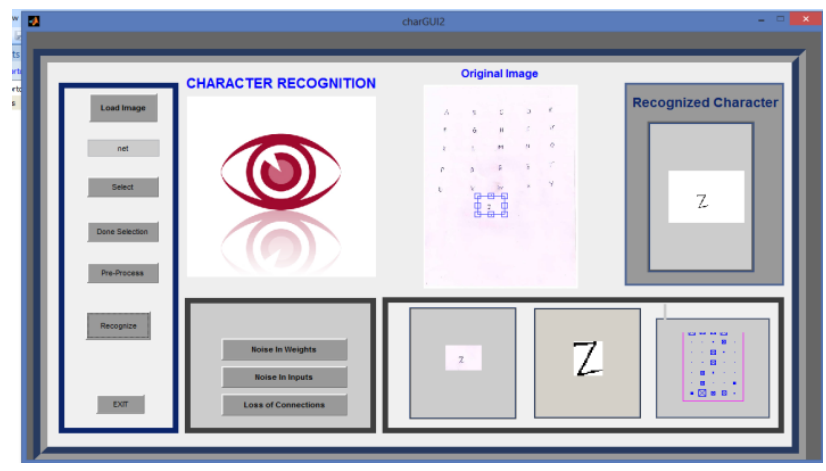

Fig 3: After recognation has complete

\section{RESULTS}

In the Back propagation algorithm the target output vector is stored which is identity matrix of size $26 \times 26$. An error goal of 0.01 was taken. The network was trained for maximum 1000 epochs. After training the network was capable of recognizing all the characters correctly. For Back Propagation Algorithm, the range of $\%$ increase of weights, for which does work properly is $0.1886-0.1422$ applicable.

6.1 Effect of Noise in Inputs: if at one time, when the noise is introduced through the noisy medium in input data, then by applying algorithm that generalizes itself, give the correct input.

6.2 Effect of Noise in Weights: Back Propagation Algorithm, When 0.3(range) random numbers are added to the weight matrices of all layers, characters are recognized correctly if at one time, noise is introduced in weights then by the back propagation network that has a capability to generalize itself to give the correct input even when it is getting noisy or distorted input.

6.3 Loss of Connection: In the network, there are three sets of weights. First connecting input to neurons of input layer. Second connecting input layer neuron to the hidden layer neurons. Third connecting hidden layer neurons to output layer neurons. If the weights connecting input to neurons of input layer, neurons of input layer to neurons of 5 hidden layer and neurons of hidden layer to all the neurons of 3 output layer( $=$ no. of classes $)$ are removed then also the network recognizes all the characters correctly. If connection of input neuron's to all the output neuron is removed and the pixel corresponding to that neuron number is off than it no difference. But if that pixel is on, in the output that becomes off.

\section{REFERENCES}

[1] NOHAJ, Miroslav, and Rudolf JAKS̆A. "Image preprocessing for optical character recognition using neural networks." Prediction of multidimensional economic data in Civilization computer game.... 30 Dávid Chochol, Ing. Rudolf Jakša, PhD. Construction of self learning system able to recognize visual information....... 44 Jozef `Corba, prof. Ing. Peter Sincák, CSc. Multiagent Adaptive Fuzzy Control of LEGO Robots. 64 
[2] Kosbatwar, S. P., and S. K. Pathan. "Pattern Association for character recognition by Back-Propagation algorithm using Neural Network approach."International Journal of Computer Science \& Engineering Survey 3.1 (2012): 127-134

[3] Sharma, Ankit, and Dipti R. Chaudhary. "Character Recognition Using Neural Network."

[4] Gupta, Amit Kumar, and Yash Pal Singh. "Analysis of Back Propagation of Neural Network Method in the String Recognition."

[5] Mamedov, Fakhreddin, and Jamal Fathi Abu Hasna. "Character Recognition using Neural Networks." IC-AI. 2006.

[6] Tezazu, Bireda. "NEURAL NETWORK IMPLEMENTATION OF CHARACTER RECOGNITION."

[7] Hong, Weibin, Wei Chen, and Rui Zhang. "The Application of Neural Network in the Technology of Image Processing." Proceedings of the International MultiConference of Engineers and Computer Scientists. Vol. 1. 2009.
[8] LeCun, Yann, et al. "Backpropagation applied to handwritten zip code recognition." Neural computation 1.4 (1989): 541-551.

[9] Jain, Anil K., Jianchang Mao, and K. Moidin Mohiuddin. "Artificial neural networks: A tutorial." Computer 29.3 (1996): 31-44.

[10] Barve, Sameeksha. "Optical Character Recognition Using Artificial Neural Network." International Journal of Advanced Research in Computer Engineering \& Technology (IJARCET) 1.4 (2012): pp-131.M. Shell. (2002)

[11] Koval, V., et al. "Smart license plate recognition system based on image processing using neural network." Intelligent Data Acquisition and Advanced Computing Systems: Technology and Applications, 2003. Proceedings of the Second IEEE International Workshop on. IEEE, 2003.

[12] Egmont-Petersen, Michael, Dick de Ridder, and Heinz Handels. "Image processing with neural networks - a review." Pattern recognition 35.10 (2002): 2279-2301. 